\title{
Collateral Circulation of the Brain \\ -With Special Reference to Atherosclerosis of the Major Cervical and Cerebral Arteries-
}

\author{
Masakuni KAMEyAMA \\ The Third Department of Internal Medicine, Faculty \\ of Medicine, University of Tokyo \\ Yokufukai Geriatric Hospital, Tokyo
}

Collateral circulation is of most concern in the pathologic process of the brain, particularly in the occlusive disease of the cervical and cerebral arteries. The collateral circulation to the brain exists in three main areas (Kaplan) $)^{1)}$ (1) through extracranial arterial connections; (2) through developmental extracranial to intracranial persisting arteries; and (3) through intracranial cerebral arterial anastomoses. The author directed efforts toward an analysis of collateral circulation to the brain of the aged subjects with some degree of atherosclerosis in the cervical as well as in the cerebral arteries. The present study consists of three parts: (1) a study on atherosclerosis of the major cervical and cerebral arteries; (2) a demonstration of the collateral circulation between the internal carotid, external carotid, and vertebral arteries; as to the intracranial anastomoses, emphasis was laid on the variations of the basal cerebral arteries, including the circle of Willis; and (3) a clinical consideration of cases with severe stenosis of the cervical arteries, with regard to cerebral vascular insufficiency due to a drop of the systemic blood pressure.

\section{Material and Method}

Four hundred autopsied cases, aged sixty and over, were selected at random at the Yokufukai Geriatric Hospital, from 1956 to 1961. In routine autopsies, the carotid and vertebral systems were removed in their entire length. The internal carotid artery was divided by anatomical convenience into three parts: (1) bifurcation, 
(2) cervical portion, and (3) terminal portion. The vertebral artery was divided into two parts: bifurcation and cervical portion. The degree of atherosclerosis was determined in each arterial segment according to the maximal stenosis. Stenosis was measured by a planimeter on histological preparations as follows:

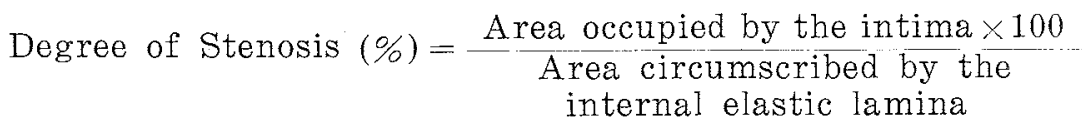

The demonstration of arterial anastomoses was performed by anatomical and/or radiographical methods. A lead salt suspension (Schlesinger) $^{2}$ of $50 \mathrm{ml}$. was injected into the common carotid in situ, at autopsy, after ligating the internal carotid; then a routine radiography was performed. The brain was carefully removed from the skull and was hardened in a $10 \%$ formol solution for ten days or more, then cut into slices. The sliced brain, dehydrated in alcohol series, was examined by a soft X-ray method (Softex).

\section{Results and Comments}

1) The subclavian and common carotid artery

As an anomalous branching, 5 cases with the right subclavian artery branching off from the aortic arch and 5 cases with the left common carotid from the right innominate artery, were observed. In these cases, atherosclerosis was more marked on the left sided arteries.

The inside diameter of the common carotid and subclavian arteries at the proximal portions ranged from $4 \mathrm{~mm}$. to $10 \mathrm{~mm}$. in the former, and from $4 \mathrm{~mm}$. to $13 \mathrm{~mm}$. in the latter. The left side was usually more narrowed than the right.

In the common carotid, the severest narrowing was found at the proximal and the distal one third of the artery. Complete occlusion due to atherosclerosis was not observed.

A case with total occlusion in the proximal portion of the left subclavian artery was encountered. In such a case, a reversal of blood flow through the cerebral artery may occur and the blood supply to the upper extremity will be via the collateral circulation through the brain, as was demonstrated by Reivich et al. ${ }^{3}$ In our 
case, however, an episode of cerebral ischemia was not observed.

2) Internal carotid and vertebral system

In the internal carotid system, cervical portion was rarely involved with atherosclerosis. In this segment of the artery, the

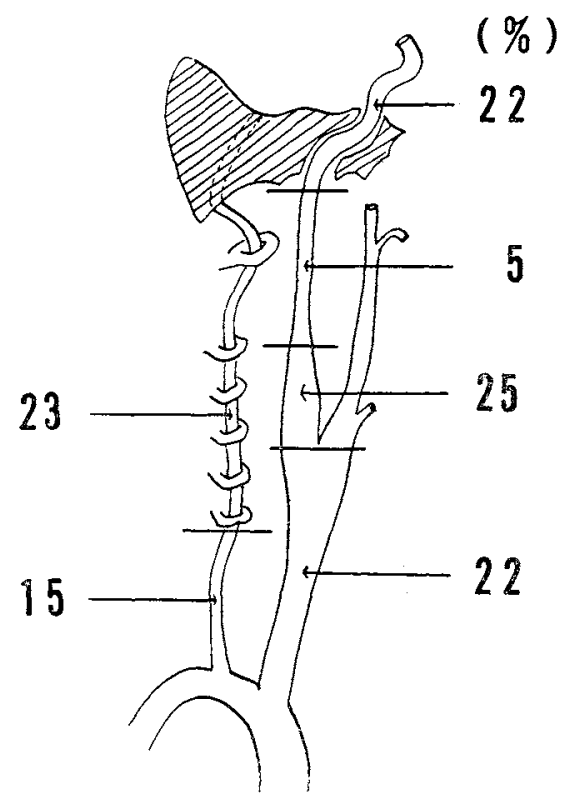

Fig. 1. Incidence of stenosis of more than $50 \%$ in the major cervical arteries ( 400 cases). muscular layer was well developed, being changed into a fibrotic tissue in the aged subjects.

The incidence of stenosis over $50 \%$ in at least one of the major cervical arteries was shown in Fig. 1. Except for the cervical portion of the internal carotid, the incidence was distributed between 15 and $25 \%$ of the examined cases.

Complete occlusion of the internal carotid artery was observed in 9 cases $(2 \%), 2$ of which died of cerebral infarction, the remaining 7 cases showing no gross findings in the brain. Complete occlusion of the vertebral artery was found in 24 cases $(6 \%)$, in only 4 of which the brain stem lesions were observed; the remaining 20 cases showed no gross findings in the brain stem.

Three groups were classified according to the grade of maximal stenosis of the internal carotid artery: (1) less than $25 \%$, (2) 25 to $50 \%$, and (3) more than $50 \%$. The incidence of cerebral infarc-

Table 1A. Degree of Stenosis of the Internal Carotid and Incidence of Cerebrovascular Lesions

\begin{tabular}{l|c|c|c}
\hline \multicolumn{1}{|c|}{ Degree of stenosis } & $\begin{array}{c}\text { less than } 25 \% \\
(129)\end{array}$ & $\begin{array}{c}25-50 \% \\
(164)\end{array}$ & $\begin{array}{c}\text { more than } 50 \% \\
(107)\end{array}$ \\
\hline Normal brains & $33 \%$ & $18 \%$ & $17 \%$ \\
Cerebral infarction & 33 & 65 & 54 \\
Cerebral hemorrhage & 23 & 31 & 25 \\
\hline
\end{tabular}


Table 1B. Degree of Severity of Cerebral Atherosclerosis and Incidence of Cerebrovascular Lesions

\begin{tabular}{l|c|c|c}
\hline Degree of atherosclerosis & -- \pm & + & $\mathbb{H}^{*}$ \\
\hline Normal brains & $57 \%$ & $22 \%$ & $0.3 \%$ \\
Cerebral infarction & 13 & 39 & 63 \\
Cerebral hemorrhage & 13 & 24 & 39 \\
\hline
\end{tabular}

*_: no atherosclerosis; $\pm:$ thin plaques involving only a small part of vessel with stenosis less than $25 \%$; + : plaques involving less than half the vessel with stenosis 25-50\%; \#: thick plaques involving more than half the vessel with stenosis more than $50 \%$.

This classification was mainly due to severity of atherosclerosis of both the middle cerebral and the basilar arteries.

tion was not paralleled by the degree of stenosis of the internal carotid, but was correlated significantly to the severity of cerebral atherosclerosis, as shown in Table 1. It is, consequently, concluded that atherosclerosis of the cerebral arteries was more important than that of cervical arteries as an etiologic factor of cerebral infarction.

3) Collateral circulation of the brain

a) Extracranial portion

Anastomoses were demonstrated between the nasal artery (a distal branch of the ophthalmic artery) and the facial arteries (a distal branch of the external carotid) by angiography performed during the autopsies. Anastomoses between the occipital artery (a branch of the external carotid) and the muscular branches of the vertebral arteries were also demonstrated in some cases. Radiopaque material injected into the external carotid, the internal carotid previously ligated, was definitely ascertained in the terminal portion of the internal carotid, anterior cerebral, middle cerebral, posterior cerebral, basilar and even in the vertebral arteries. The material was found in both sides of the meningeal arteries. In the cases with proliferated arachnoid villi, the radiopaque material was found penetrating into the cerebral cortex through that proliferation. Of the anastomoses between the external and internal carotid, the one via the ophthalmic artery was most prominent. Radiographic examination of the sliced brain showed the dye in the leptomeningeal arteries and in the subcortical white matter, as well 


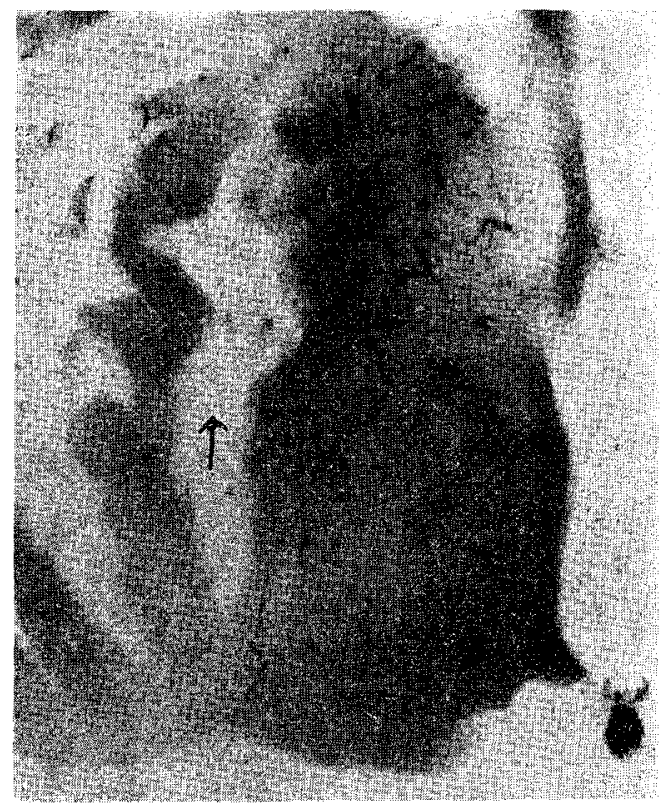

Fig. 2. Radiopaque material in the sliced brain (soft X-ray method). Arrow: infarction.

as in the basal ganglia (Fig. 2).

The ophthalmic artery usually showed no sclerotic changes throughout the course in the optic nerve; while in the proximal portion stenosis was not uncommon, due to intimal thickening with fibroelastosis.

The degree of stenosis of the external and internal carotid, at the bifurcation, and that of the vertebral arteries were significantly paralleled $(\mathrm{P}<0.01)$ (Table 2 ).

Considering the above-mentioned data, it is conceivable that the function of the collateral circulation of the brain is diminished in aged subjects, in spite of an existence of anastomoses demonstrated anatomically and/or angiographically.

b) Intracranial portion

The most important intracranial anastomoses of the cerebral artery are those of the leptomeningeal arteries on the surface and the circle of Willis at the base. The present paper laid an emphasis on the clinicopathologic significance of the variations of the basal cerebral arteries, including the circle of Willis. 
Table 2. Correlation of Degree of Stenosis between Internal Carotid, External Carotid, and Vertebral Artery

\begin{tabular}{c|c|c|c|c|c|c}
\hline $\begin{array}{c}\text { Internal } \\
\text { carotid }\end{array}$ & \multicolumn{3}{|c|}{ External carotid } & \multicolumn{3}{|c}{ Vertebral } \\
\hline $\begin{array}{c}\text { less than } \\
25 \%\end{array}$ & $25-50 \%$ & $\begin{array}{c}\text { more than } \\
50 \%\end{array}$ & $\begin{array}{c}\text { less than } \\
25 \%\end{array}$ & $25-50 \%$ & $\begin{array}{c}\text { more than } \\
50 \%\end{array}$ \\
\hline $\begin{array}{c}\text { less than } \\
25 \%\end{array}$ & 111 & 33 & 14 & 90 & 88 & 37 \\
\hline \begin{tabular}{c}
$25-50 \%$ \\
\hline $\begin{array}{c}\text { more than } \\
50 \%\end{array}$
\end{tabular} & 22 & 55 & 21 & 28 & 54 & 38 \\
\hline 15 & 23 & 35 & 11 & 22 & 32 \\
\hline
\end{tabular}

As variations of the basal cerebral arteries, marked caliber difference, abnormal branching of the arteries, and absence of or string-like patent communicating arteries were investigated. In 140 cases (35\%), marked variations were not observed in the basal cerebral arteries (normal type).

Concerning the incidence of variations, a significant difference was found between the 167 cases with cerebral infarction (infarction group) and 90 cases without cerebrovascular lesions. $\Delta s$ in Table 2, marked caliber difference between the right and left side of the anterior cerebral and that of the vertebral arteries was respectively observed in significantly higher incidence in the infarction group $(\mathrm{P}<0.01)$. String-like posterior communicating artery

Table 3. Variations of the Basal Cerebral Arteries

\begin{tabular}{l|c|c|c} 
& $\begin{array}{c}\text { Normal brains } \\
(90)\end{array}$ & $\begin{array}{c}\text { Cerebral infaction } \\
(167)\end{array}$ & \\
\hline $\begin{array}{l}\text { No variations } \\
\text { Marked right and left } \\
\text { caliber difference of the } \\
\text { anterior cerebral }\end{array}$ & $52 \%$ & $22 \%$ & $\mathrm{p}<0.01$ \\
$\begin{array}{c}\text { Marked right and left } \\
\text { caliber difference of the } \\
\text { vertebral }\end{array}$ & 14 & 28 & $\mathrm{p}<0.05$ \\
$\begin{array}{c}\text { Absent or string-like } \\
\text { posterior communicating } \\
\text { artery }\end{array}$ & 7 & 45 & $\mathrm{p}<0.01$ \\
$\begin{array}{c}\text { Embryonic posterior } \\
\text { cerebral }\end{array}$ & 18 & 13 & $\mathrm{p}<0.2$ \\
$\begin{array}{c}\text { Primitive type } \\
-\end{array}$ & 9 & 29 & $\mathrm{p}<0.05$ \\
\hline
\end{tabular}


or even absence of this artery was observed in $13 \%$ of the infarction group and in $7 \%$ of the normal brain group; the difference, hewever, was not statistically significant.

The variation of an embryonic derivation of the posterior cerebral artery from the internal carotid was observed in $38.1 \%$ of the total numbers, the incidence of which is two or three times higher than that of both Americans and Europeans. According to Alpers et $a 1 .{ }^{4}$, the incidence of this variation was in $14.6 \%$ of 350 cases, and Mitterwallner ${ }^{5}$ reported that the incidence was $19 \%$ of 360 cases.

It is suggested that there may be some form of heredity in the formation of this variation. This variation was classified into four types as follows (Fig. 3):
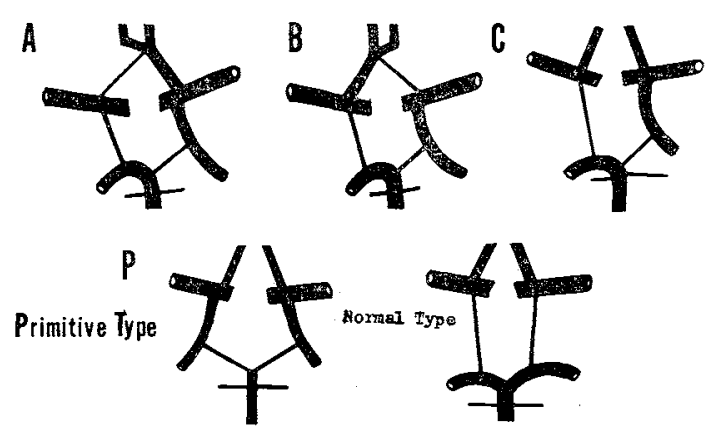

Fig. 3. Four types of the variation with embryonic posterior cerebral artery.

A type: the anterior cerebral artery was markedly larger on the same side of embryonic derivation of the posterior artery from the internal carotid.

$B$ type: the anterior cerebral artery was markedly smaller on the same side of embryonic derivation of the posterior artery.

C type: the anterior cerebral arteries were normal.

Primitive type: bilateral posterior cerebral arteries branched off from the internal carotid.

The incidence of cerebral infarction was most frequent in A type (93\%), followed by B, C, primitive type and normal type (Table 4).

Why did the variation groups show a higher incidence of cerebrovascular lesions? The reason may be explained, in part, by the 
Table 4. Types of Variations with Embryonic Posterior Cerebral Artery and Incidence of Cerebrovascular Lesions

\begin{tabular}{c|c|c|c|c} 
Type & No. of cases & Normal brains & $\begin{array}{c}\text { Cerebral } \\
\text { infarction }\end{array}$ & $\begin{array}{c}\text { Cerebral } \\
\text { hemorrhage }\end{array}$ \\
\hline A & 14 & $0 \%$ & $93 \%$ & $50 \%$ \\
B & 19 & 11 & 47 & 26 \\
C & 80 & 16 & 40 & 24 \\
Primitive type & 40 & 20 & 30 & 30 \\
Normal type & 104 & 45 & 33 & 19 \\
\hline
\end{tabular}

fact that in the variation groups a sufficiently collateral circulation was not formed in occlusive diseases of the internal carotid, vertebral, and cerebral arteries, in addition to the fact that atherosclerosis was more marked in the variation groups, as reported previously by the author. ${ }^{\text {b) }}$

4) Severe stenosis of the cervical arteries and blood pressure

The degree of stenosis of the four major cervical arteries was averaged in each individual, the distribution of which was presented in Fig. 4. Stenosis more than 50\% was found in 67 (17\%) of 400 cases.

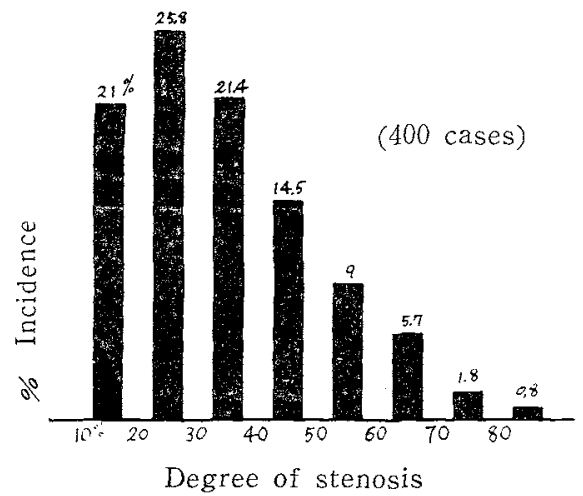

Fig. 4. Frequency distribution of cases with averaged stenosis more than $50 \%$ in the major cervical arteries.

Subjects with stenosis over 50\% had shown moderate or severe hypertension in the clinical course. Hypertension of these cases was regarded as a compensatory physiologic factor to maintain as adequate cerebral circulation as possible, including collateral circulation. Indeed, in 38 of the 67 cases, cerebral vascular insufficiency (CVI) was manifested recurrently, along with fall of blood pressure. 
The value of the systolic blood pressure obtained during the attack was shown in Fig. 5, compared with that of normal conditions. As presented there, the value was markedly varied. In elderly subjects, therefore, it is impossible to determine the bottom level of the systolic blood pressure to be safe from an episode of CVI; the margin of a drop of the blood pressure is thought to be important. The value of the margin obtained in 38 cases averaged $76 \pm 24 \mathrm{~mm}$. $\mathrm{Hg}$. Cerebrovascular insufficiency was frequently observed in febrile conditions of various causes.

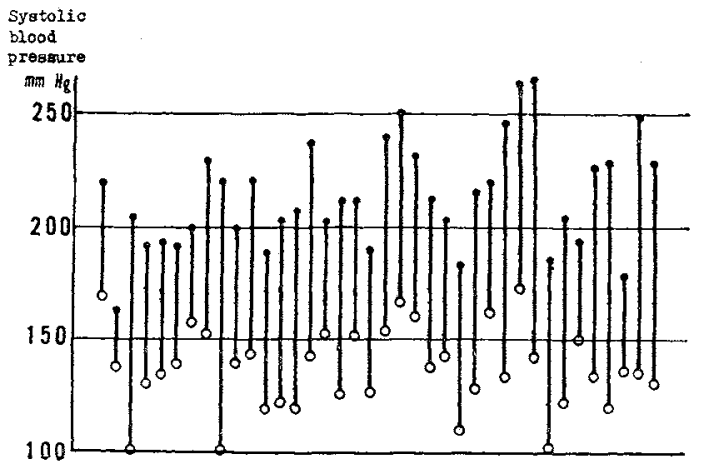

Fig. 5. Cerebral vascular insufficiency and blood pressure in 38 cases with severe stenosis in the cervical arteries. : systolic blood pressure in normal conditions; $O$ : systolic blood pressure of the same subjects at the onset of an episode of CVI. Margin of a drop was averaged $76 \pm 24 \mathrm{~mm}$. $\mathrm{Hg}$.

\section{General Comment}

The relational atherosclerosis of the cervical arteries to cerebrovascular diseases is an impending problem in Japan, as well as in western countries. The author investigated severity of atherosclerosis in the major cervical and cerebral arteries of the aged subjects from a clinicopathological standpoint. Occlusion of the internal carotid artery was observed in $2 \%$ of the cases during routine autopsies. Fisher ${ }^{7}$ found the internal carotid occlusion in 28 of 432 cases, Haltquist ${ }^{8}$ in 91 of 3500 cases, Samuel ${ }^{9}$ in 3 of 82 cases, and Martin et al. ${ }^{10)}$ in 9 out of 100 cases.

Our percentage was lower than those of these western authors. In the vertebral arteries, thoroughly examined, the incidence of 
complete occlusion was $6 \%$ in our series. It is, however, remarkable that the large number of patients with cervical arterial occlusion showed no gross findings in the brain at autopsy. This fact should call attention to the importance of interpreting arteriographic evidence ef stenosis or occlusion in the light of specific localization of origin of the clinical symptoms. On this point, atherosclerosis of the cerebral arteries is considered more important as an etiologic factor of cerebral infarction, than that of cervical arteries. In other words, occlusion of the major cervical arteries is not necessarily responsible for the neurological manifestations and cerebral lesions in the aged subjects.

Anastomoses between the internal carotid, external carotid, and vertebral arteries were demonstrated by anatomical and/or radiographical method. However, severity of atherosclerosis of these arteries was significantly correlated each to the others, and stenotic changes of the distal branches of these arteries were markedly advanced in many cases; therefore, sufficient collateral circulation to the brain might not be expected. In the aged brain, arterial anastomoses through the proliferated arachnoid villi were demonstrated, with surprisingly little contribution to the principal cerebral circulation.

In the intracranial portion, leptomeningeal arteries have many anastomoses on the surface of the brain, as shown by Vander Eecken. ${ }^{11)}$ At the base, the circle of Willis is regarded as the most important in the collateral circulation. Circle anomaly, however, is not uncommon, as already has been pointed out by many workers. ${ }^{12}$ Variations of the basal cerebral arteries including the circle of Wills were found in $65 \%$ of our series. This presentation, consequently, laid an emphasis on an analysis of clinicopathologic significance of the variations. Marked right and left caliber difference of the anterior cerebral and of the vertebral arteries was observed in higher incidence in cases with cerebral infarction than in cases without gross findings in the brain. Embryonic branching of the posterior cerebral artery from the internal carotid was also more common in the infarction groups. This type of variation was two or three times more frequent in Japanese than in Americans and in Europeans. This variation was divided into four groups 
according to existing or non-existing caliber difference of the anterior cerebral artery. Of the four groups, Type A was most frequently involved with cerebral infarction. In the primitive type the incidence of vascular lesions was nearly equally matched with that of the normal type. It is conceivable that a symmetric configuration of the arterial tree is favorable for maintaining normal cerebral circulation. Considering from a clinicopathological standpoint, variation groups were placed at a disadvantage in forming collateral circulation according to their characteristic vascular pattern with super-imposed atherosclerosis.

Blood pressure is most important to maintain normal cerebral circulation. In cases with severe stenosis of the neck arteries, even a slight drop of the blood pressure may cause serious clinical states. Indeed, the margin of fluctuation of the blood pressure to maintain physiological cerebral circulation was much diminished in these subjects. Cerebral vascular insufficiency (CVI) which recurred in 38 of 67 cases with severe stenosis of the neck arteries, was induced with a drop of the blood pressure. Because of marked individual difference, it is thought to be unreasonable to determine the critical level of the blood pressure as a cause of CVI. The margin of fluctuation of the blood pressure is most important clinically; in an average, a drop of $76 \pm 24 \mathrm{~mm}$. Hg. systolic, was obtained in 38 cases with attacks of CVI. CVI was observed frequently in febrile conditions of various causes. In elderly patients, much attention should be paid to the blood pressure level, especially in febrile conditions.

\section{Summary and Conclusion}

Collateral circulation of the brain was studied on 400 autopsied cases aged 60 and over, selected at random, with special reference to atherosclerosis of the cervical and cerebral arteries.

1) Of the arteries branching off from the aortic arch, the lumen of the subclavian and the common carotid was more narrowed on the left side of the body than on the right.

2) Fifteen to $25 \%$ of 400 cases examined showed stenosis over $50 \%$ in one or more of the internal carotid and vertebral arteries. 
Complete occlusion was observed in $9(2 \%)$ and in $24(6 \%)$ cases in the internal carotid and vertebral arteries, respectively; however, in only 2 of the former and 4 of the latter, cerebral infarction was confirmed at autopsy.

3) The incidence of cerebral infarction was not paralleled by the degree of stenosis of the internal carotid, but was significantly correlated to the severity of cerebral atherosclerosis. It was, consequently, concluded that the cerebral infarction was due more to stenotic changes of the cerebral arteries than to those of the cervical arteries. This fact may be important from a clinical point of vicw in order to interpret angiographic findings.

4) The grade of stenosis of the internal carotid was significantly correlated to that of the vertebral as well as of the external carotid arteries.

5) Anastomoses between the external carotid, the internal carotid, and the vertebral arteries were demonstrated by anatomical and/or radiographical methods. The function of collateral circulation of these anastomoses, however, might not sufficiently be expected in the aged subjects, with already advanced sclerotic changes in these arterial parts.

6) The variations of the basal cerebral arteries were observed in $65 \%$ of 400 cases. In the cases with cerebral infarction, the incidence of the right and left caliber difference of the anterior and of the vertebral arteries was significantly higher than in the cases without gross findings in the brain. The embryonic derivation of the posterior cerebral artery from the internal carotid was also found more commonly in the cases with cerebral infarction. The latter type of variation was two or three times more frequent in Japanese than in Americans and in Europeans. An existence of a type of heredity was suggested in the formation of this variation. Clinicopathologic significance of the variations of the basal cerebral arteries was discussed with special emphasis on collateral circulation.

7) Thirty-eight of 67 cases with severe stenosis in the cervical arteries showed recurrent episodes of CVI along with a drop of the blood pressure; in an average, a drop of $76 \pm 24 \mathrm{~mm}$. Hg, systolic. CVI was frequently observed in febrile conditions with various causes. 
The author wishes to thank Professor S. Okinaka, M. D. (University of Tokyo) and F. Amako, M. D. (Medical Chief of the Yokufukai Geriatric Hospital) for their advice and encouragement.

\section{References}

1) Kaplan, H. A.: Collateral circulation of the brain. Neurology 11(4), Part 2:9, 1961.

2) Schlesinger, M. J.: An injection plus dissection study of coronary artery occlusions and anastomoses. Am. Heart J. 15: 528, 1938.

3) Reivich, M., Holling, H. E., Roberts, B., and Toole, J.F.: Reversal of blood flow through the vertebral artery and its effect on cerebral circulation. New Engl. J. Med. 265: 878, 1961.

4) Alpers, B. J., Berry, R. G., and Paddison, R. M.: Anatomical studies of the circle of Willis in normal brain. A. M. A. Arch. Neurol. \& Psychiat. 81: 409, 1959.

5) Mitterwallner, Fr. V.: Variationsstatistische Untersuchungen an den basalen Hirngefaessen. Acta anat. 24:51, 1955.

6) Kameyama, M.: A clinicopathologic study on variations of the basal cerebral arteries. Recent Advance in Research of the Nervous System 5: 758, 1961.

7) Fisher, M.: Occlusion of the carotid artery: Further experiences. A. M. A. Arch. Neurol. \& Psychiat. 72: 187, 1954.

8) Haltquist, G. T.: Ueber Thrombose und Embolie der Arteria carotis und hierbei vorkommende Gehirnstoerungen. Jena, Gustav Fisher Verlag, 1942. Cited by Fisher, M. ${ }^{7}$

9) Samuel, K.C.: Atherosclerosis and occlusion of the internal carotid artery. J. Path. \& Bact. $71: 391,1956$.

10) Martin, M. J., Whisnant, J. P., and Sayre, G.P.: Occlusive vascular disease in the extracranial cerebral circulation. A. M. A. Arch. Neurol. 5: 530, 1960.

11) Vander Becken, H.: Anastomoses between the leptomeningeal arteries of the brain. Their morphological, pathological and clinical significance. Springfield, I11. Charles C: Thomas, 1959, pp. 36-62.

12) Krayenbueh1, H., and Yasargil, M. G.: Die vasculaeren Erkrankungen im Gebiet der Arteria vertebralis und Arteria basilaris. Stuttgart, Georg Thieme Verlag, 1957, pp. 36-50. 